\title{
ARTICLE
}

Clinical Study

\section{Association between single-nucleotide polymorphisms and adverse events in nivolumab-treated non-small cell lung cancer patients}

Sander Bins ${ }^{1}$, Edwin A. Basak¹, Samira el Bouazzaoui ${ }^{2}$, Stijn L. W. Koolen ${ }^{1}$, E. Oomen - de Hoop ${ }^{1}$, Cor H. van der Leest ${ }^{3}$, Astrid A. M. van der Veldt ${ }^{1}$, Stefan Sleijfer ${ }^{1}$, Reno Debets ${ }^{1}$, Ron H. N. van Schaik ${ }^{2}$, Joachim G. J. V. Aerts ${ }^{4}$ and Ron H. J. Mathijssen ${ }^{1}$

BACKGROUND: Treatment with PD-1 inhibitors can be hampered by severe auto-immune-related toxicities. Our objective was to identify single-nucleotide polymorphisms (SNPs) in genes previously associated with auto-immunity, which are associated with toxicities in nivolumab-treated NSCLC patients. This was in order to identify patients prone to develop severe toxicities and to gain more insight into the underlying pathobiology.

METHODS: We analysed 322 nivolumab-treated patients and assessed the association with toxicities for seven SNPs in four genes, which are considered contributors to PD-1-directed T-cell responses, i.e., PDCD1, PTPN11, ZAP70 and IFNG. Every SNP was tested for its association with toxicity endpoints. Significant associations were tested in a validation cohort.

RESULTS: A multivariable analysis in the exploration cohort showed that homozygous variant patients for PDCD1 804C $>T$ ( $r$ 2227981) had decreased odds for any grade treatment-related toxicities ( $n=96 ;$ OR $0.4 ; 95 \% \mathrm{Cl} 0.2-1.0 ; p=0.039$ ). However, this result could not be validated ( $n=85$; OR $0.9 ; 95 \% \mathrm{Cl} 0.4-1.9 ; p=\mathrm{NS}$ ).

CONCLUSIONS: Our results show that it is unlikely that the investigated SNPs have a clinical implication in predicting toxicity. A finding, even though negative, that is considered timely and instructive towards further research in biomarker development for checkpoint inhibitor treatments.

British Journal of Cancer (2018) 118:1296-1301; https://doi.org/10.1038/s41416-018-0074-1

\section{INTRODUCTION}

Tumours are able to escape from an effective immune response by exploiting phenotypical and functional changes in tumour cells as well as stromal cells that compromise infiltration, migration and local activation of anti-tumour T lymphocytes. ' One of these changes, representing a dominant escape mechanisms in some tumours, is over-expression of the immune checkpoint programmed cell death protein 1 (PD-1) by tumour-infiltrating CD8positve $T$ cells, and can be counteracted by nivolumab, thereby rescuing exhausted $T$ cells from a non-functional status. ${ }^{2,3}$ Nivolumab is an anti-PD-1 antibody that is, among others, approved for the treatment of melanoma, non-small cell lung cancer (NSCLC), renal cell carcinoma, microsatellite instability-high colorectal carcinoma, and squamous cell carcinoma of the head and neck. ${ }^{4-9}$ Approval for other indications is expected to follow in the near future. Unfortunately, the application of anti-PD-1 blocking agents can be accompanied by severe toxicities, such as dermatitis, hypothyroidism, colitis and pneumonitis. These auto-immune-related adverse events can have extensive consequences, such as treatment discontinuation. Markers to identify patients who are at risk to develop such toxicities are key to develop a more personalised treatment approach.

Germline genetic aberrations are frequently associated with systemic toxicities as a consequence of anti-tumour agents. ${ }^{10}$ Hence, we argue that variants of genes involved in the PD-1 pathway are associated with the occurrence of PD-1-inhibitorinduced auto-immune-related toxicities. Moreover, PD-1, its downstream proteins SHP2 and ZAP70, and IFNY (Fig. 1) ${ }^{11-13}$ are already known to be involved in disorders of the immune system: single-nucleotide polymorphisms (SNPs) in their encoding genes (PDCD1, PTPN11, ZAP70 and IFNG) have been associated with autoimmune syndromes ${ }^{14-18}$ and with excessive inflammation. ${ }^{19}$ For example, SNPs in PDCD1 are associated with ankylosing spondylitis and diabetes mellitus type 1, SNPs in ZAP70 are associated with Crohn's disease and SNPs in PTPN11 are associated with a more severe course of inflammation after gastric infection and ulcerative colitis. Not every patient with a predisposing genotype develops an auto-immune phenotype, but the sensitivity of the PD-1 axis, or its activity, might be altered in those persons. When treated with PD-(L)1 inhibiting drugs, these (asymptomatic)

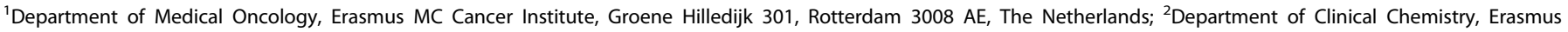

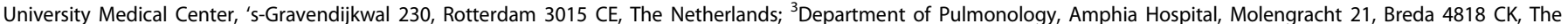
Netherlands and ${ }^{4}$ Department of Pulmonology, Erasmus University Medical Center, 's-Gravendijkwal 230, Rotterdam 3015 CE, The Netherlands

Correspondence: Ron H. J. Mathijssen (a.mathijssen@erasmusmc.nl)

These authors contributed equally: Sander Bins, Edwin A. Basak.

This work was presented in part as a proffered paper at the 4th ESMO Symposium on Immunology (November 2016, Lausanne, Switserland).

Received: 3 January 2018 Revised: 9 March 2018 Accepted: 13 March 2018

Published online: 26 April 2018 
a

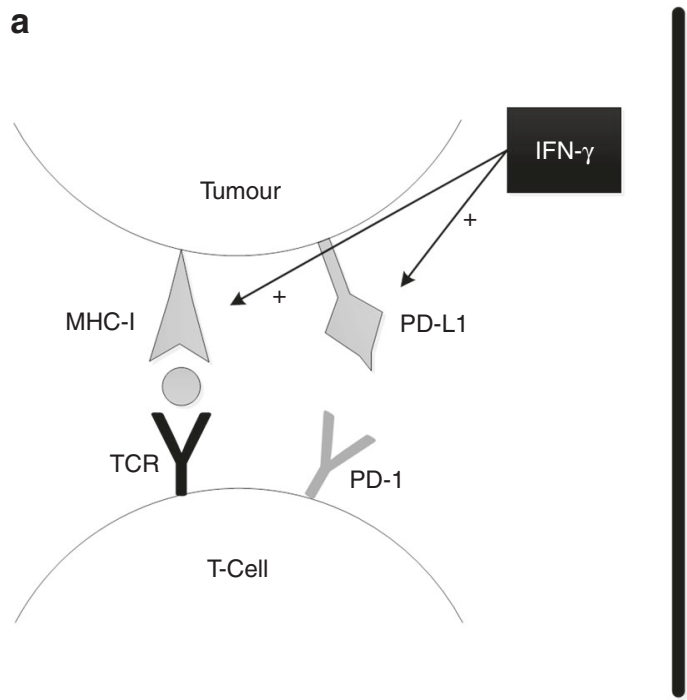

b

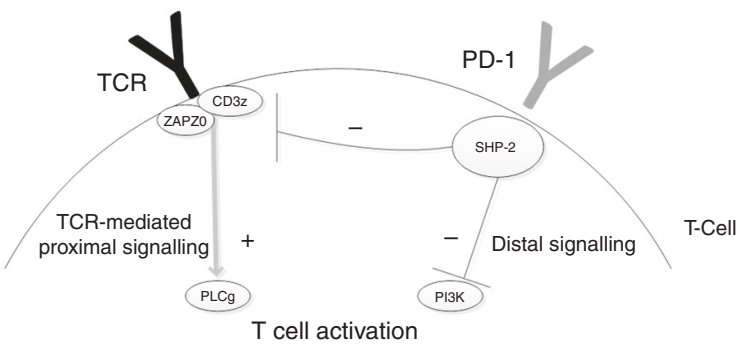

Fig. 1 a Interaction between a tumour cell and a $\mathrm{T}$ cell. Tumour cells can activate $\mathrm{T}$ cells by presenting an antigen through major histocompatibility complex (MHC) to the T cell receptor (TCR). Under influence of interferon-gamma (IFN $\gamma$ ) tumours can express ProgrammedDeath ligand-1 (PD-L1), which inhibits TCR signalling by binding and activating Programmed-Death-1 (PD-1) expressed by T cells. b Proximal PD-1 pathway signalling. Activated PD-1 recruits SHP2, which inhibits ZAP70 function. ZAP70 is an important protein in the signalling pathway of the TCR. Complementary to its effect on ZAP70, SHP2 may also inhibit PI3K upon PD-1 activation. Both effects lead to inhibition of T cell activation. Note: the PD-1 pathway comprises many more proteins and signal transduction pathways, but these are omitted from this figure since they are not included in our analysis

carriers of an aberrant genotype might be more prone to develop immune related adverse events than patients with a wildtype PD1 axis. Hence, we hypothesised that additional PD-1 inhibition by nivolumab would trigger auto-immunity and consequently lead to more toxicity in patients who harbor germline genetic polymorphisms in the PD-1 axis. In this study, we explored a large cohort of nivolumab-treated NSCLC patients in daily clinical practice and studied whether patients with SNPs in the PD-1 and PD-1-related genes experienced toxicity more (or less) frequently or severely than other patients.

\section{MATERIALS AND METHODS}

\section{Study design}

We collected data from NSCLC patients who started nivolumab monotherapy treatment, without other concomitant anti-cancer agents (e.g., chemotherapy), at two large Dutch hospitals (the Erasmus MC Cancer Institute, Rotterdam, and at the Amphia Hospital, Breda) between July 26th 2013 and April 5th 2017. Clinical data was collected until June 1st 2017. Patients from whom whole blood for DNA analysis was (prospectively) collected were included in this study (local ethics board study number MEC 02-1002). Patients were ranked based on date of treatment start, thereafter patients were alternately allocated to an exploration cohort or a validation cohort, each consisting of 161 patients.

Patient characteristics were collected from the hospitals electronic patient record systems and included demographic and clinical information (e.g., age at start of treatment, gender, ethnicity, WHO performance status at start of treatment, previous anti-tumour treatments, treatment interruptions, NSCLC subtype and toxicities). WHO performance status was determined by judgment of the clinician at the nearest time point before start of nivolumab treatment, and was regarded as "missing" if this point was more than 1 month before treatment start. Concomitant use of oral or intravenous corticosteroids to resolve immune-related toxicities was also recorded and regarded as a surrogate for adverse events.

Adverse events from start of treatment until end of follow-up were retracted from the patient status, and best corresponding grade was retrieved according to National Cancer Institute Common Terminology Criteria for Adverse Events (NCl-CTCAE) v4.03 if not already graded appropriately in the patient status. Adverse events that were possibly, probably, or definitely related to the nivolumab treatment were classified as treatmentrelated adverse events. The diagnosis of hepatitis was based on judgment of the treating clinician. An adverse event was considered pre-existent if it was present in the same or higher degree before treatment start, and therefore not considered as an event.

Selection of SNPS

We selected seven SNPs in the PDCD1, PTPN11, ZAP70 and IFNG genes for analysis (see Table 1 for details). SNPs with a reported minor allele frequency (MAF) above $5 \%$ were included.

DNA isolation

Four hundred microliters of whole-blood specimens were collected in EDTA tubes and DNA was extracted in a final elution volume of $200 \mu \mathrm{L}$ using the MagNAPure Compact instrument (Roche Diagnostics GmbH, Mannheim, Germany) and the Nucleic Acid Isolation Kit I (Roche Diagnostics $\mathrm{GmbH}$ ).

Taqman genotyping

Genotyping was performed using predesigned DME Taqman allelic discrimination assays on the Life Technologies Taqman 7500 system (Applied Biosystems, Life Technologies Europe BV, Bleiswijk, The Netherlands; Table 1). Each assay consisted of two allele-specific minor groove binding (MGB) probes, labeled with the fluorescent dyes VIC and FAM. Polymerase chain reactions (PCR) were performed in a reaction volume of $10 \mu \mathrm{L}$, containing assay-specific primers, allele-specific Taqman MGB probes (Applied Biosystems), Abgene Absolute QPCR ROx Mix (Thermo Scientific, Life Technologies Europe BV, Bleiswijk, The Netherlands) and genomic DNA $(20 \mathrm{ng})$. The thermal profile consisted of 40 cycles of denaturation at $95^{\circ} \mathrm{C}$ for $20 \mathrm{~s}$, annealing at $92^{\circ} \mathrm{C}$ for $3 \mathrm{~s}$ and extension at $60^{\circ} \mathrm{C}$ for $30 \mathrm{~s}$. Genotypes were scored by measuring allele-specific fluorescence using the 7500 software v2.3 for allelic discrimination (Applied Biosystems). 
Table 1. Investigated single-nucleotide polymorphisms

\begin{tabular}{|c|c|c|c|c|c|c|c|c|c|c|c|}
\hline Gene & Protein & rs-number & Variant & Assay ID & Cohort & WT & HET & HVAR & Undetermined & MAF & $\mathrm{HWE}^{\mathrm{a}}$ \\
\hline \multirow[t]{6}{*}{ PDCD1 } & PD-1 & rs2227981 & $804 C>T$ & c_57931286_20 & Exploration & 50 & 76 & 34 & 1 & $45 \%$ & 0.61 \\
\hline & & & & & Validation & 44 & 78 & 39 & 0 & $48 \%$ & 0.70 \\
\hline & & rs2227982 & $644 C>T$ & c_57931287_10 & Exploration & 159 & 2 & 0 & 0 & $1 \%$ & 0.9 \\
\hline & & & & & Validation & 161 & 0 & 0 & 0 & $0 \%$ & $\mathrm{n} / \mathrm{a}$ \\
\hline & & rs10204525 & $* 889 \mathrm{G}>\mathrm{A}$ & c__172862_10 & Exploration & 139 & 20 & 2 & 0 & $7 \%$ & 0.21 \\
\hline & & & & & Validation & 132 & 27 & 2 & 0 & $10 \%$ & 0.65 \\
\hline \multirow[t]{2}{*}{ PTPN11 } & SHP2 & rs2301756 & $333-223 A>G$ & c__2978562_20 & Exploration & 131 & 30 & 0 & 0 & $9 \%$ & 0.19 \\
\hline & & & & & Validation & 128 & 30 & 3 & 0 & $11 \%$ & 0.43 \\
\hline \multirow[t]{2}{*}{ ZAP70 } & ZAP70 & rs 13420683 & $-21-4127 C>A$ & c__1278468_10 & Exploration & 89 & 55 & 13 & 4 & $26 \%$ & 0.29 \\
\hline & & & & & Validation & 88 & 51 & 20 & 2 & $29 \%$ & 0.007 \\
\hline \multirow[t]{4}{*}{ IFNG } & $\mathrm{IFN} \gamma$ & rs2069718 & $367-895 C>T$ & c_15799728_10 & Exploration & 57 & 74 & 30 & 0 & $42 \%$ & 0.49 \\
\hline & & & & & Validation & 55 & 74 & 30 & 2 & $42 \%$ & 0.57 \\
\hline & & rs2069705 & $-1616 \mathrm{~T}>\mathrm{C}$ & c_15944115_20 & Exploration & 72 & 70 & 19 & 0 & $34 \%$ & 0.75 \\
\hline & & & & & Validation & 73 & 65 & 23 & 0 & $34 \%$ & 0.18 \\
\hline
\end{tabular}

WT wildtype, HET heterozygous, HVAR homozygous variant, MAF minor allele frequency, HWE Hardy-Weinberg equilibrium. ${ }^{\text {a }}$ If $<0.05$ not consistent with $H W E$

Statistics

Distribution of genotypes was tested for the Hardy-Weinberg equilibrium (HWE) using the $X^{2}$ test (Table 1). Since ZAP70-32$4127 \mathrm{C}>\mathrm{A}$ was not in HWE in the validation cohort, this SNP was excluded from further analyses. SNPs with a MAF $<5 \%$ in our cohort were also excluded from further analyses, which was the case for PDCD1 644C>T. Linkage disequilibrium (LD) analyses were performed using SNAP. ${ }^{20}$ Because none of the analysed SNP pairs met our preset criteria for LD (R2 >0.8), all SNPs were analysed individually. For every SNP, the best fitting model (that is, the model resulting in the best association) was selected from four models, i.e., a dominant, recessive, additive, and multiplicative model. ${ }^{21}$ The dominant and recessive models were used to test associations between SNPs and toxicity, steroid use, and temporary or definitive treatment discontinuation due to toxicity using the $x^{2}$ test or, in case one of the observed numbers was $\leq 10$ or one of the expected numbers was $<5$, with Fisher's exact test. The additive and multiplicative models were used to test the SNPs in logistic regression as ordinal and linear predictor variables, respectively. If a SNP was associated with toxicity with $p<0.1$, it was entered in a multivariable logistic regression model together with age and gender in order to avoid bias by those parameters. Multivariable analysis was only performed in case of approximately 10 or more events per assessed variable in order to avoid bias of the regression coefficients. ${ }^{22}$ Models with a significant outcome in univariable or multivariable analysis in the exploration cohort were examined in the validation cohort. SPSS software v21 (SPSS, Chicago, IL, USA) was used for the above-mentioned analyses. A two-sided $p<0.05$ was regarded as significant and no correction for multiple testing was applied.

\section{RESULTS}

Patient characteristics

Blood samples for DNA analysis were available for 322 NSCLC patients in total, of whom $63 \%(n=202)$ were male, having a mean age at start of therapy of 65 years, and $58 \%(n=188)$ had a WHO performance status of 1 at start. More baseline characteristics for both the exploration and validation cohorts are depicted in Table 2. All characteristics were comparable between both groups ( $t$-test). All patients were treated with the standard dose of nivolumab at 3 $\mathrm{mg} / \mathrm{kg}$ Q2W that was not changed during treatment. Median absolute starting dose was $222 \mathrm{mg}$ (IQR 192-263 mg) and median duration of follow-up was 126 days (IQR 70-214 days).

\begin{tabular}{|c|c|c|}
\hline & $\begin{array}{l}\text { Number of patients ( } n \\
=161 ; \text { exploration } \\
\text { cohort) }\end{array}$ & $\begin{array}{l}\text { Number of patients } \\
\text { ( } n=161 ; \text { validation } \\
\text { cohort) }\end{array}$ \\
\hline \multicolumn{3}{|l|}{ Gender } \\
\hline Male & $108(67 \%)$ & $94(58 \%)$ \\
\hline Female & 53 (33\%) & $67(42 \%)$ \\
\hline \multicolumn{3}{|l|}{ Age at start (years) } \\
\hline Mean $( \pm S D)$ & $64( \pm 8.1)$ & $65( \pm 9.1)$ \\
\hline \multicolumn{3}{|c|}{ WHO performance status } \\
\hline 0 & $26(16 \%)$ & $28(17 \%)$ \\
\hline 1 & 95 (59\%) & $93(58 \%)$ \\
\hline 2 & $2(1 \%)$ & $5(3 \%)$ \\
\hline 3 & $1(1 \%)$ & $0(0 \%)$ \\
\hline Unknown & $37(23 \%)$ & $35(22 \%)$ \\
\hline \multicolumn{3}{|l|}{ Primary lung tumour } \\
\hline Adenocarcinoma & $97(60 \%)$ & $101(63 \%)$ \\
\hline $\begin{array}{l}\text { Squamous cell } \\
\text { carcinoma }\end{array}$ & $47(29 \%)$ & $49(30 \%)$ \\
\hline Great cell carcinoma & $15(9 \%)$ & $7(4 \%)$ \\
\hline Unspecified & $2(1 \%)$ & $4(3 \%)$ \\
\hline \multicolumn{3}{|c|}{ Number of pre-treatment lines } \\
\hline 0 & $1(1 \%)$ & $0(0 \%)$ \\
\hline 1 & $104(65 \%)$ & $113(70 \%)$ \\
\hline 2 & $44(27 \%)$ & 38 (24\%) \\
\hline$>2$ & $12(7 \%)$ & $10(6 \%)$ \\
\hline \multicolumn{3}{|l|}{ Ethnicity } \\
\hline Caucasian & $152(94 \%)$ & $156(97 \%)$ \\
\hline Other & $4(3 \%)$ & $1(1 \%)$ \\
\hline Unknown & $5(3 \%)$ & $4(3 \%)$ \\
\hline
\end{tabular}

Adverse events

Fifty patients $(16 \%)$ had any grade $\geq 3$ toxicity (Table 3 and Supplementary Table S1). When analysing all-grade toxicity that was not pre-existent, hypothyroidism or hyperthyroidism $(n=129$; $40 \%)$, elevated transaminases $(n=106 ; 33 \%)$, and skin toxicity 
Table 3. Frequencies of toxicity endpoints corrected for pre-existence

Number of patients Number of $(n=161$; exploration patients $(n=161$; cohort) validation cohort)

\begin{tabular}{|c|c|c|}
\hline \multicolumn{3}{|l|}{ Any grade toxicity } \\
\hline Diarrhea & $6(4 \%)$ & $7(4 \%)$ \\
\hline Skin toxicity & $23(14 \%)$ & $23(14 \%)$ \\
\hline Elevated transaminases & $50(31 \%)$ & $56(35 \%)$ \\
\hline Elevated bilirubin & $7(4 \%)$ & $7(4 \%)$ \\
\hline Hepatitis & $6(4 \%)$ & $2(1 \%)$ \\
\hline $\begin{array}{l}\text { Hypothyroidism or } \\
\text { hyperthyroidism }\end{array}$ & $64(40 \%)$ & $65(40 \%)$ \\
\hline $\begin{array}{l}\text { Pneumonitis/Interstitial } \\
\text { lung disease }\end{array}$ & $8(5 \%)$ & $8(5 \%)$ \\
\hline Colitis & $2(1 \%)$ & $4(3 \%)$ \\
\hline $\begin{array}{l}\text { Rheumatological } \\
\text { toxicity }\end{array}$ & $10(6 \%)$ & $13(8 \%)$ \\
\hline Hypophysitis & $1(1 \%)$ & $1(1 \%)$ \\
\hline $\begin{array}{l}\text { Treatment-related } \\
\text { toxicity }\end{array}$ & $96(60 \%)$ & $85(53 \%)$ \\
\hline \multicolumn{3}{|l|}{ Other outcomes } \\
\hline $\begin{array}{l}\text { Decreased renal } \\
\text { clearance grade } \geq 2\end{array}$ & $28(17 \%)$ & $41(26 \%)$ \\
\hline $\begin{array}{l}\text { Any grade } 3 \text { or higher } \\
\text { toxicity }\end{array}$ & $24(15 \%)$ & $26(16 \%)$ \\
\hline $\begin{array}{l}\text { Any grade } 3 \text { or higher } \\
\text { treatment-related } \\
\text { toxicity }\end{array}$ & $14(9 \%)$ & $9(6 \%)$ \\
\hline Steroid use & $42(26 \%)$ & $38(24 \%)$ \\
\hline \multicolumn{3}{|c|}{ Treatment stop caused by toxicity } \\
\hline Temporary interruption & $15(9 \%)$ & $15(9 \%)$ \\
\hline $\begin{array}{l}\text { Definitive } \\
\text { discontinuation }\end{array}$ & $6(4 \%)$ & $5(3 \%)$ \\
\hline
\end{tabular}

( $n=46 ; 14 \%)$ occurred most frequently. Treatment-related grade $\geq 3$ toxicities occurred in 23 patients $(7 \%)$. Any grade treatmentrelated adverse events occurred in 181 patients (56\%; Supplementary Table S2).

Eighty patients $(25 \%)$ required oral or intravenous steroids during nivolumab treatment. Thirty patients (9\%) temporarily interrupted nivolumab treatment because of toxicities, whereas eleven patients $(3 \%)$ had to permanently stop treatment because of toxicities.

Treatment details

At the end of the data collection period, 150 patients (47\%) were still being treated with nivolumab. A total of 42 patients (13\%) stopped because of clinical deterioration, 108 patients (34\%) stopped after progressive disease (assessed by the treating clinician), eleven patients (3\%) stopped because of treatmentrelated toxicities and ten patients (3\%) stopped because of other reasons, i.e., due to patients' request $(n=5)$, sudden death $(n=4)$ or a non-measurable lesion after re-evaluation of the baseline CTscan $(n=1)$. Twenty-three patients $(7 \%)$ were lost to follow-up.

Association of SNPs with toxicity during nivolumab treatment All outcomes of the exploration cohort that occurred in a sufficient number of patients for univariable analysis are shown in Supplementary Table S3. Those outcomes with an association of $p<0.1$ in relation to the concerning SNP are described in Table 4. The PTPN11 333-223A>G and PDCD1 ${ }^{*} 889 \mathrm{G}>\mathrm{A}$ SNPs were only tested in a dominant model since the number of homozygous mutant patients was too low for reliable analysis. Since skin toxicity, rheumatological toxicity, any grade $\geq 3$ toxicity, treatment related grade $\geq 3$ toxicity, and temporary stop caused by toxicity occurred in an insufficient number of patients for reliable multivariable analysis, these outcomes were only analysed univariably. Diarrhea, elevated bilirubin, hepatitis, pneumonitis, neuropathy, colitis and permanent stop caused by toxicity were ineligible for univariable analysis. Decreased renal clearance ( $\geq$ grade 2 ), elevated transaminases, hypothyroidism or hyperthyroidism and treatment-related adverse events (any grade) were studied in multivariable analyses.

In the exploration cohort, homozygous variant patients for PDCD1 804C $>$ T had decreased odds for developing any grade treatment-related adverse events (OR $0.4 ; 95 \% \mathrm{Cl} 0.2-1.0 ; p=$ 0.039). At least one variant allele at PTPN11 333-223A $>G$ was associated with increased odds for elevated transaminases (OR $2.4 ; 95 \% \mathrm{Cl} 1.1-5.5 ; p=0.036$ ). The relationship between hypothyroidism or hyperthyroidism and PTPN11 333-223A $>\mathrm{G}$ in univariable analysis did not hold in multivariable analysis (OR 0.4 ; $95 \% \mathrm{Cl} 0.2-1.0 ; p=0.052$ ). Of the endpoints that could only be analysed univariably due to the number of patients, IFNG $-1616 \mathrm{~T}>\mathrm{C}$ was associated with rheumatological toxicity (OR 6.0; 95\% Cl 1.5-23.9; $p=0.019$ ).

Associations between SNPs and endpoints that showed significance when tested in the exploration cohort, were retested in the validation cohort of which results are shown in Table 5. Since the associations between PTPN11 333-223A >G and elevated transaminases (OR 1.509; 95\% Cl 0.690-3.300; $p=0.301$ ), and between PDCD1 $804 \mathrm{C}>\mathrm{T}$ and any grade treatment-related adverse events (OR 0.923; 95\% Cl 0.449-1.899; $p=0.828$ ) had $p$ values $>0.1$, these were not considered for multivariable analysis in the validation cohort. Furthermore, the above described association between IFNG $-1616 \mathrm{~T}>\mathrm{C}$ and rheumatological toxicity in univariable analysis did not hold in the validation cohort (OR $0.5 ; 95 \% \mathrm{Cl} 0.1-3.9 ; p=0.695)$.

\section{DISCUSSION}

Although this is - to the best of our knowledge-the largest pharmacogenetic pathway analysis in nivolumab treated NSCLC patients so far, we could not validate SNPs of the PD-1 or PD-1related genes relevant for toxicity. Nonetheless, in the exploration cohort, the TT genotype in the PDCD1 804C>T SNP (rs2227981) was associated with less nivolumab related toxicity. Moreover, at least one $G$ allele in the PTPN11 333-223A>G SNP (rs2301756) was associated with an increased risk for developing elevated transaminases. Also, in univariable analysis, homozygous mutant patients for the IFNG -1616T>C SNP had higher odds for developing rheumatological toxicity. However, all findings could not be replicated in the validation cohort.

Interestingly, a variant allele of PDCD1 $804 C>T$ has previously been associated with susceptibility for ankylosing spondylitis and type I diabetes. ${ }^{14}$ Possibly, this could be related to a decreased activity profile of the PD-1 pathway in patients harboring this SNP, and these patients may therefore experience less inhibition of $T$ cell activation (Fig. 1). It may seem paradoxical that harboring two variant alleles in the rs2227981 SNP is associated with less toxicity in the exploration cohort, whereas earlier findings relate it to a higher incidence of auto-immune related events. Speculating on this, it could be that patients with the variant allele already have less PD-1-mediated T cell inhibition, which may make this pathway less susceptible to blockade by nivolumab. Consequently, these patients may experience fewer treatment-related toxicities than wildtype patients.

Our finding in the exploration cohort that patients with at least one variant allele in the rs2301756 SNP have an increased risk for developing elevated transaminases is in line with earlier studies: the variant allele has previously been associated with 
Table 4. Association between SNPs with $p<0.1$ in the univariable analysis and endpoints (exploration cohort)

\begin{tabular}{|c|c|c|c|c|c|c|}
\hline \multirow[t]{2}{*}{ Endpoint } & \multirow[t]{2}{*}{ Factor } & \multirow[t]{2}{*}{ Genotype } & \multicolumn{2}{|l|}{ Univariable } & \multicolumn{2}{|l|}{ Multivariable } \\
\hline & & & OR $(95 \% \mathrm{Cl})$ & $p$-value & OR $(95 \% \mathrm{Cl})$ & $p$-value \\
\hline \multirow[t]{3}{*}{ Decreased renal clearance ( $\geq$ grade 2 ) } & Age & & & & $1.056(0.999-1.116)$ & 0.056 \\
\hline & Gender (f. vs. m.) & & & & $1.364(0.555-3.350)$ & 0.498 \\
\hline & $P D C D 1804 C>T$ & $\mathrm{CC} \rightarrow \mathrm{CT} \rightarrow \mathrm{TT}^{\mathrm{a}}$ & $1.631(0.917-2.903)$ & $0.096^{\mathrm{b}}$ & $1.545(0.864-2.763)$ & 0.142 \\
\hline \multirow[t]{3}{*}{ Elevated transaminases (any grade) } & Age & & & & $1.010(0.967-1.055)$ & 0.649 \\
\hline & Gender (f. vs. m.) & & & & $1.741(0.841-3.604)$ & 0.135 \\
\hline & PTPN11 333-223A>G & $G G+A G$ vs. $A A$ & $2.309(1.024-5.208)$ & $0.041^{\mathrm{c}}$ & $2.421(1.061-5.523)$ & 0.036 \\
\hline \multirow{3}{*}{$\begin{array}{l}\text { Hypothyroidism or hyperthyroidism (any } \\
\text { grade) }\end{array}$} & Age & & & & $0.989(0.950-1.030)$ & 0.598 \\
\hline & Gender (f. vs. m.) & & & & $1.158(0.579-2.315)$ & 0.678 \\
\hline & PTPN11 333-223A>G & $\mathrm{GG}+\mathrm{AG}$ vs. $\mathrm{AA}$ & $0.395(0.158-0.985)$ & $0.061^{d}$ & $0.403(0.161-1.007)$ & 0.052 \\
\hline \multirow[t]{3}{*}{ Treatment-related adverse events (any grade) } & Age & & & & $1.007(0.967-1.049)$ & 0.738 \\
\hline & Gender (f. vs. m.) & & & & $1.234(0.609-2.498)$ & 0.560 \\
\hline & $P D C D 1804 C>T$ & TT vs. $C C+C T$ & $0.454(0.211-0.978)$ & $0.041^{c}$ & $0.440(0.202-0.958)$ & 0.039 \\
\hline Rheumatological toxicity (any grade) & IFNG $-1616 \mathrm{~T}>\mathrm{C}^{\mathrm{e}}$ & $\mathrm{CC}$ vs. TT $+\mathrm{TC}$ & $6.044(1.531-23.857)$ & $0.019^{d}$ & & \\
\hline
\end{tabular}

$O R$ odds ratio, $\mathrm{Cl}$ confidence interval, $f$. vs. $m$. females versus males. ${ }^{a}$ Additive model was used. ${ }^{b}$ Logistic regression was used. ${ }^{\mathrm{C}} \mathrm{Chi}-\mathrm{square}$ test was used. ${ }^{d}$ Fisher's Exact test was used. ${ }^{\text {e}}$ Only tested univariably due to the number of events

Table 5. Association between SNPs and endpoints with a significant correlation in the exploration cohort

\begin{tabular}{|c|c|c|c|c|}
\hline \multirow[t]{2}{*}{ Endpoint } & \multirow[t]{2}{*}{ Factor } & \multirow[t]{2}{*}{ Genotype } & \multicolumn{2}{|l|}{ Univariable } \\
\hline & & & OR $(95 \% \mathrm{Cl})$ & $p$-value \\
\hline Elevated transaminases (any grade) & PTPN11 333-223A>G & $G G+A G$ vs. $A A$ & $1.509(0.690-3.300)$ & $0.301^{\mathrm{a}}$ \\
\hline Treatment-related adverse events (any grade) & $P D C D 1804 C>T$ & TT vs. $\mathrm{CC}+\mathrm{CT}$ & $0.923(0.449-1.899)$ & $0.828^{\mathrm{a}}$ \\
\hline Rheumatological toxicity (any grade) & IFNG $-1616 \mathrm{~T}>\mathrm{C}$ & $\mathrm{CC}$ vs. $\mathrm{TT}+\mathrm{TC}$ & $0.477(0.059-3.857)$ & $0.695^{\mathrm{b}}$ \\
\hline
\end{tabular}

susceptibility for ulcerative colitis in the Japanese population ${ }^{18}$ and with an increased risk for gastric atrophy after $H$. Pylori infection, ${ }^{19}$ possibly caused by chronic inflammation. Our finding supports the notion that genetic variants in the SHP2 gene (PTPN11) prevent T cell suppression and do result in stronger T cell reactions, in particular when there is additional inhibition of PD-1 via nivolumab treatment. Speculating on the underlying biological mechanism, we argue that reduced SHP2 activity - in case of the above-mentioned SNP-results in less de-phosphorylation (and thereby de-activation) of its targets ZAP-70 and PI3K. As such, the 'brake' on proximal TCR-mediated signalling as well as more distal $\mathrm{PI} 3 \mathrm{~K}$ signalling is relieved, which then results in enhanced $\mathrm{T}$ cell activation (see Fig. 1).

In addition, the earlier reported finding between SNPs in IFNG with systemic lupus erthythaematosis ${ }^{17}$ corresponds with our finding in the exploration cohort, in which this SNP is associated with rheumatological toxicities. The rs2069718 SNP in IFNG, and the rs10204525 SNP in PDCD1, located on two genes that affect each other's expression according to a negative feedback loop, were not associated with outcomes in both cohorts, possibly due to the influence of other adjacent pathways related to IFNY and PD-1. We did not explore these adjacent pathways in the current analysis, however, this research is part of ongoing work.

The explorative nature of this study and the retrospective data collection may result in a slightly different low-grade toxicity profile than reported previously. ${ }^{23}$ Nevertheless, the clinically most relevant endpoints, i.e., any grade $\geq 3$ toxicity and treatmentrelated grade $\geq 3$ toxicity, are unlikely to be underrepresented in this study as the median follow-up time ( $>4$ months) exceeds the previously described expected time for toxicities to occur. ${ }^{24}$ In fact an endpoint such as treatment-related adverse events eliminates distortion of the association between a SNP and an endpoint by excluding adverse events that could be a consequence of other factors, such as disease progression. Furthermore, some endpoints, e.g., colitis, occurred too infrequent to be assessed in multivariable analysis, which requires about ten patients per assessed variable. ${ }^{22}$ The cutoff date resulted in a relatively short follow-up time in a small number of patients in both cohorts $(n=$ 13 versus $n=12$, respectively). However, earlier trials described that most relatively frequent adverse events occur during the first few weeks of treatment, such as skin, gastrointestinal, and hepatic toxicities. ${ }^{25}$ Therefore, we expect that inclusion of the concerning patients had a negligible effect on most important endpoints.

Associations between SNPs and survival are also important, since predictive biomarkers for survival are currently lacking. As the survival data are not mature enough yet, we will assess these associations once the data allow us to. Although germline genetics might seem logical biomarkers for general systemic effects, such as adverse events, and although (local) antitumour T cell effects are also affected by somatic mutations and mutational load, ${ }^{26}$ prior treatment, and possibly even the microbiome, ${ }^{27}$ SNPs studied here might provide additional predictive information to these other biomarkers.

Despite our above stated explanations for the findings in the exploration cohort, it should be emphasised that they could not be validated, even though the distribution of events per genotype was comparable between the two cohorts for the relationships 
between elevated transaminases and the SNP in PTPN11 and between any grade treatment-related adverse events and the SNP in PDCD1 804. However, in the validation cohort, there was one patient with a rheumatological toxicity in the homozygous mutant group for the IFNG -1616T>C SNP, which could explain the contradictory finding with the exploration cohort.

The current genotyping effort should not be considered comprehensive. Even though it is likely that other factors than germline genetics may contribute to the occurrence of adverse events, our study provides a starting point from where other genes, or even other SNPs in the investigated genes, could be investigated towards future biomarker development for anti-PD-1 treatment outcomes.

As mentioned, main limitations of this analysis arise from the retrospective data collection. Therefore, subtle adverse events (grade 2 or less) may not have been mentioned by the treating physician, or its pre-existence may not have been noticed. Also, the distinction between treatment-related toxicities or adverse events caused by other sources may be unclear for some lower-grade and general toxicities. Moreover, since some toxicities occur only in a very small proportion of immunotherapy treated patients, these outcomes had to be excluded from the analysis. Finally, this analysis was restricted to a limited proportion of the PD- 1 pathway and its associated genes and polymorphisms.

In conclusion, this study shows that future biomarker research for nivolumab treatment outcomes can and should embrace germline genetics. However, it is unlikely that the investigated SNPs have a clinical implication in the prediction of toxicity in NSCLC patients treated with nivolumab. Considering underlying biological mechanisms, these SNP probably have no relevance in patients with other tumour types and patients receiving other anti-PD-1 agents. The reported lack of associations facilitates further research in biomarker development for checkpoint inhibitor treatments.

\section{AUTHOR CONTRIBUTIONS}

R.M. declares that he had full access to the data in the study and final responsibility for the decision to submit for publication. S.B., E.B., C.H.L., J.A. and R.M. were involved in recruiting patients and collecting data. Genetic analyses were performed by S.E.B. and R.H.N.S. Statistical analyses were performed by S.B. and E.B. and were supervised by E.O.H., statistician. All authors were involved in study design, data interpretation and manuscript writing

\section{ADDITIONAL INFORMATION}

Supplementary information is available for this paper at https://doi.org/10.1038/ s41416-018-0074-1.

Competing interests: Van der Leest received personal fees from BMS. Van der Veldt is a member of an advisory board at BMS. Aerts is a member of an advisory board at BMS and has patents pending for analysis of biomarkers for immunotherapy. Mathijssen has patents pending for analysis of biomarkers for immunotherapy. The remaining authors declare no competing interests.

Ethical approval: The study was approved by the local ethics board of the Erasmus Medical Center study as number MEC 02-1002. The study was performed in accordance with the Declaration of Helsinki.

Availability of data and materials: Data supporting the results are archived in the Erasmus Medical Center.

Note: This work is published under the standard license to publish agreement. After 12 months the work will become freely available and the license terms will switch to a Creative Commons Attribution 4.0 International licence (CC BY 4.0).

\section{REFERENCES}

1. Debets, R., Donnadieu, E., Chouaib, S. \& Coukos, G. TCR-engineered T cells to treat tumours: Seeing but not touching? Semin. Immunol. 28, 10-21 (2016).

2. Freeman, G. J. et al. Engagement of the PD-1 immunoinhibitory receptor by a novel B7 family member leads to negative regulation of lymphocyte activation. J. Exp. Med. 192, 1027-1034 (2000)

3. Nishimura, H., Nose, M., Hiai, H., Minato, N. \& Honjo, T. Development of lupus-like autoimmune diseases by disruption of the PD-1 gene encoding an ITIM motifcarrying immunoreceptor. Immunity 11, 141-151 (1999).

4. Brahmer, J. R. et al. Phase I study of single-agent anti-programmed death-1 (MDX$1106)$ in refractory solid tumours: safety, clinical activity, pharmacodynamics, and immunologic correlates. J. Clin. Oncol. 28, 3167-3175 (2010).

5. Brahmer, J. et al. Nivolumab versus docetaxel in advanced squamous-cell nonsmall-cell lung cancer. N. Engl. J. Med. 373, 123-135 (2015).

6. Larkin, J. et al. Combined nivolumab and ipilimumab or monotherapy in untreated melanoma. N. Engl. J. Med. 373, 23-34 (2015).

7. Motzer, R. J. et al. Nivolumab versus everolimus in advanced renal-cell carcinoma N. Engl. J. Med 373, 1803-1813 (2015).

8. Overman, M. J. et al. Nivolumab in patients with metastatic DNA mismatch repairdeficient or microsatellite instability-high colorectal cancer (CheckMate 142): an open-label, multicentre, phase 2 study. Lancet Oncol. 18, 1182-1191 (2017).

9. Ferris, R. L. et al. Nivolumab for recurrent squamous-cell carcinoma of the head and neck. N. Engl. J. Med. 375, 1856-1867 (2016).

10. Hertz, D. L. \& Rae, J. Pharmacogenetics of cancer drugs. Annu Rev. Med. 66, 65-81 (2015).

11. Okazaki, T., Maeda, A., Nishimura, H., Kurosaki, T. \& Honjo, T. PD-1 immunoreceptor inhibits B cell receptor-mediated signalling by recruiting src homology 2domain-containing tyrosine phosphatase 2 to phosphotyrosine. Proc. Natl. Acad. Sci. USA 98, 13866-13871 (2001).

12. Sheppard, K. A. et al. PD-1 inhibits T-cell receptor induced phosphorylation of the ZAP70/CD3zeta signalosome and downstream signalling to PKCtheta. FEBS Lett. 574, 37-41 (2004).

13. Taube, J. M. et al. Colocalization of inflammatory response with B7-h1 expression in human melanocytic lesions supports an adaptive resistance mechanism of immune escape. Sci. Transl. Med. 4, 127ra137 (2012).

14. Lee, Y. H., Bae, S. C., Kim, J. H. \& Song, G. G. Meta-analysis of genetic polymorphisms in programmed cell death 1 . Associations with rheumatoid arthritis, ankylosing spondylitis, and type 1 diabetes susceptibility. Z. Rheumatol. 74, 230-239 (2015).

15. Chen, $\mathrm{S}$. et al. The associations between PD-1, CTLA-4 gene polymorphisms and susceptibility to ankylosing spondylitis: a meta-analysis and systemic review. Rheumatol. Int. 36, 33-44 (2016).

16. Bouzid, D. et al. Association of ZAP70 and PTPN6, but Not BANK1 or CLEC2D, with inflammatory bowel disease in the Tunisian population. Genet Test. Mol. Biomark. 17, 321-326 (2013)

17. Kim, K. et al. Interferon-gamma gene polymorphisms associated with susceptibility to systemic lupus erythematosus. Ann. Rheum. Dis. 69, 1247-1250 (2010).

18. Narumi, Y. et al. Polymorphisms of PTPN11 coding SHP-2 as biomarkers for ulcerative colitis susceptibility in the Japanese population. J. Clin. Immunol. 29, 303-310 (2009).

19. Hamajima, N., Naito, M., Kondo, T. \& Goto, Y. Genetic factors involved in the development of Helicobacter pylori-related gastric cancer. Cancer Sci. 97, 1129-1138 (2006).

20. Johnson, A. D. et al. SNAP: a web-based tool for identification and annotation of proxy SNPs using HapMap. Bioinformatics 24, 2938-2939 (2008).

21. Lewis, C. M. Genetic association studies: design, analysis and interpretation. Brief. Bioinform. 3, 146-153 (2002).

22. Peduzzi, P., Concato, J., Kemper, E., Holford, T. R. \& Feinstein, A. R. A simulation study of the number of events per variable in logistic regression analysis. J. Clin. Epidemiol. 49, 1373-1379 (1996).

23. Spain, L., Diem, S. \& Larkin, J. Management of toxicities of immune checkpoint inhibitors. Cancer Treat. Rev. 44, 51-60 (2016).

24. Postow, M. A. Managing immune checkpoint-blocking antibody side effects. Am. Soc. Clin. Oncol. Educ. Book 35, 76-83 (2015).

25. Borghaei, $H$. et al. Nivolumab versus docetaxel in advanced nonsquamous nonsmall-cell lung cancer. N. Engl. J. Med. 373, 1627-1639 (2015).

26. Rizvi, N. A. et al. Cancer immunology. Mutational landscape determines sensitivity to PD-1 blockade in non-small cell lung cancer. Science 348, 124-128 (2015).

27. Sivan, A. et al. Commensal Bifidobacterium promotes antitumour immunity and facilitates anti-PD-L1 efficacy. Science 350, 1084-1089 (2015). 